\title{
Method of the Year 2013
}

\author{
Methods to sequence the DNA and RNA of single cells are poised to transform many areas of \\ biology and medicine.
}

Once considered a technical challenge reserved for a few specialized labs, single-cell transcriptome and genome sequencing is becoming robust and broadly accessible. Exciting insights from recent studies are revealing the potential to understand biology at the unitary resolution of life, and last year marked a turning point in the widespread adoption of these methods to address a variety of research questions. For these reasons, single-cell sequencing is our choice of Method of the Year for 2013.

Every cell is unique-it occupies an exclusive position in space, carries distinct errors in its copied genome and is subject to programmed and induced changes in gene expression. Yet most DNA and RNA sequencing is performed on tissue samples or cell populations, in which biological differences between cells can be obscured by averaging or mistaken for technical noise.

Single-cell methods offer a way to dissect this heterogeneity. Single-cell DNA sequencing can reveal mutations and structural changes in the genomes of cancer cells, which tend to have high mutation rates. This information can be used to describe the clonal structure and to trace the evolution and spread of the disease. These approaches are also revealing a surprising level of mosaicism in somatic tissues such as the brain, the functional consequences of which will need to be elucidated in the coming years.

Differences between cells can be greater yet at the RNA level, even within seemingly uniform populations such as immune cells that have been purified on the basis of cell-surface markers. Single-cell transcriptome profiling can identify biologically relevant differences in cells, even when cells may not be distinguishable by marker genes or cell morphology, and can be used to group cells in an unbiased way.

Another advantage of single-cell sequencing is that it makes rare cells more accessible to analysis, provided that methods are available to isolate or enrich these cells from their heterogeneous environments. Cells taken from very specific spatiotemporal contexts, including microbes sampled from the environment, can be evaluated at the genome scale. In the clinic, single-cell sequencing can help with preimplantation screening of in vitro-fertilized embryos; and cancer diagnostics based on rare circulating tumor cells that can seed cancer at distant body sites becomes possible.

The central challenge of scaling down to the cellular level is capturing such a tiny amount of template and amplifying it to generate enough material for high-throughput sequencing. Maintaining fidelity and avoiding biases during heavy amplification is not trivial, but doing so is critical to ensuring adequate sequence coverage, accurate quantification and detection of sequence variation.

Recent protocol improvements and commercial offerings are helping to ease the adoption of single-cell sequencing approaches. Microfluidics and microwell technologies are also improving reproducibility and scale. We outline some basic workflows and considerations in a Primer (p. 18). In a News Feature (p. 13), Kelly Rae Chi highlights how single-cell sequencing approaches are already being effectively applied in the areas of biological development, cancer and neurobiology.

Single-cell genome sequencing reduces the sequence complexity of cell mixtures. In a Commentary (p. 19), Paul Blainey and Stephen Quake discuss how this can be leveraged to determine recombination frequencies in cells undergoing meiosis, to tease apart the maternal and paternal genomic contributions, or haplotypes, and to enable the assembly of microbial genomes sampled directly from complex mixtures in the environment.

In another Commentary, Rickard Sandberg argues that we are entering an era of single-cell transcriptome sequencing that will deepen our understanding of gene regulation and cellular transcriptional states, improve our ability to identify differences between healthy and diseased tissues, and profile rare cancerous cells (p. 22).

By focusing on genome and transcriptome sequencing, we do not mean to discount the importance of alternative single-cell approaches. Other methods such as in situ hybridization can effectively interrogate sequences in single cells in addition to providing the physical address of transcripts or DNA in intact tissue. Epigenomic profiling of single cells will add important information on gene regulation. Beyond sequence, approaches such as mass cytometry and mass spectrometry will help to characterize protein expression in single cells on a large scale. A final Commentary by James Eberwine and colleagues (p. 25) discusses the directions that such complementary technologies will need to take to understand single cells at the level of function.

We also present our Methods to Watch (p. 28), a selection of methods or areas of methodological development that we believe have particularly interesting potential in the coming years.

We hope that you enjoy our special feature. A happy 2014 to all our readers! 